\title{
Most rigid representations and Cayley index ${ }^{*}$
}

\author{
Joy Morris , Josh Tymburski \\ Department of Mathematics and Computer Science, University of Lethbridge, \\ Lethbridge, AB T1K 3M4, Canada
}

Received 27 March 2017, accepted 3 January 2018, published online 6 February 2018

\begin{abstract}
For any finite group $G$, a natural question to ask is the order of the smallest possible automorphism group for a Cayley graph on $G$. A particular Cayley graph whose automorphism group has this order is referred to as an MRR (Most Rigid Representation), and its Cayley index is a numerical indicator of this value. Study of GRRs showed that with the exception of two infinite families and thirteen individual groups, every group admits a Cayley graph whose MRR is a GRR, so that the Cayley index is 1 . The full answer to the question of finding the smallest possible Cayley index for a Cayley graph on a fixed group was almost completed in previous work, but the precise answers for some finite groups and one infinite family of groups were left open. We fill in the remaining gaps to completely answer this question.
\end{abstract}

Keywords: Cayley graph, Cayley index, GRR, MRR, automorphisms.

Math. Subj. Class.: 05C25

\section{Introduction}

All groups and graphs in this paper are finite. All of our graphs are simple, undirected, and have no loops.

A Cayley graph $\Gamma=\operatorname{Cay}(G, S)$ where $S \subseteq G$ with $S=S^{-1}$ and $1 \notin S$, is the graph whose vertices are the elements of $G$, with $(g, g s) \in E(\Gamma)$ if and only if $g \in G$ and $s \in S$. We refer to $S$ as the connection set for $\Gamma$. Let $A=\operatorname{Aut}(\Gamma)$. Observe that $L_{G}$, the left-regular representation of $G$, lies in $A$, so $|G|$ divides $|A|$.

* The authors would like to thank the anonymous referee whose careful reading and generous advice helped us to correct some errors and historical inaccuracies, and improved the exposition of this paper.

This research was supported in part by the Natural Science and Engineering Research Council of Canada, Grant RGPIN-2011-238552.

E-mail addresses: joy.morris@uleth.ca (Joy Morris), josh.tymburski@uleth.ca (Josh Tymburski) 
Definition 1.1. The Cayley index $c(\Gamma)$ of the Cayley graph $\Gamma=\operatorname{Cay}(G, S)$, is $\left|A: L_{G}\right|$. The Cayley index $c(G)$ of the group $G$ is $\min _{S \subseteq G, S=S^{-1}} c(\operatorname{Cay}(G, S))$; that is, the lowest Cayley index of any Cayley graph on the group $G$.

Definition 1.2. A Cayley graph $\Gamma=\operatorname{Cay}(G, S)$ is a GRR (Graphical Regular Representation) for $G$ if $c(\Gamma)=1$.

Thus, groups that admit GRRs are precisely the groups whose Cayley index is 1 . In order to completely characterise these groups, we require another definition.

Definition 1.3. Let $A$ be an abelian group of even order, and $y$ an involution in $A$. Then the generalised dicyclic group $\operatorname{Dic}(A, y)$ is $\langle A, x\rangle$ where $x \notin A, x^{2}=y$, and $x^{-1} a x=a^{-1}$ for every $a \in A$.

Notice that under this definition, the generalised dicyclic group $\operatorname{Dic}(A, y)$ will be abelian if and only if $A$ is an elementary abelian 2-group.

The study of GRRs involved many researchers and papers. Some of the most influential work along the way appeared in [6, 7,9]. Watkins [16] observed that there are two infinite families of graphs that cannot admit GRRs: generalised dicyclic groups, and abelian groups that are not elementary abelian 2-groups. Imrich [7] resolved the problem for abelian groups by classifying the elementary abelian 2-groups, finding exactly three that admit no GRR. Watkins in a series of papers, some with coauthor Nowitz [11, 12, 16, 17, 18], discovered ten nonabelian groups that admit no GRRs. Hetzel [5] proved that aside from the two infinite families noted by Watkins, and the thirteen small solvable groups (of order at most 32) found by Imrich, Nowitz, and Watkins, every solvable group admits a GRR. Godsil [3] showed that every non-solvable group admits a GRR.

In the case where a group fails to admit a GRR, a natural question to ask is: what is the Cayley index of the group, and what is a Cayley graph on the group that has that Cayley index? The following terminology was coined in [10].

Definition 1.4. Let $G$ be a group with $c(G)>1$, and let $\Gamma=\operatorname{Cay}(G, S)$ be a Cayley graph on $G$ with $c(\Gamma)=c(G)$. Then we say that $\Gamma$ is an MRR (Most Rigid Representation) for $G$.

The bulk of this paper is divided into 4 sections. In Section 2, we describe the groups that do not admit a GRR but do not lie in either of the infinite families of groups that do not admit a GRR. For each of these groups, we find its Cayley index and an MRR. In Section 3, we find the Cayley index of every abelian group, and find MRRs for those groups whose Cayley index is greater than 2. In Section 4, we consider a subfamily of generalised dicyclic groups (specifically, the hamiltonian 2-groups), and show that the smallest two of these have Cayley index 16, while the rest have Cayley index 8. Finally, in Section 5, we find the Cayley index for every generalised dicyclic group that was not included in Section 4.

Much of the work that we summarise in this paper was done in [10], but the authors of [10] left some gaps. Our paper fills all of these gaps, thus completing their work. Specifically, we fill the following gaps. We examine the Cayley indices of the groups that do not lie in either of the infinite families; we give the Cayley indices for the four abelian groups for which they did not specify it (although they stated that these had been found by computer); we find the precise Cayley index for generalised dicyclic groups of order at most 96 (they bounded almost all of these by 4 , but most in fact have Cayley index 2); and we find the 
Cayley indices for all hamiltonian 2-groups (they bounded these by 16, but almost all have Cayley index 8). Table 1 summarises this work, providing the Cayley index for every finite group.

For a number of the small individual groups, we found MRRs using Sage [15] and its GAP package [14]. The Cayley index of any of the graphs we present can be easily checked via computer, using this or other appropriate software.

Throughout this paper,

$$
Q_{8}=\left\{ \pm 1, \pm i, \pm j, \pm k: i j=k=-j i, i^{2}=j^{2}=k^{2}=-1\right\}
$$

is the usual representation of the quaternion group of order 8 . We use $D_{2 n}$ for $n \geq 3$ to represent the dihedral group of order $2 n$. Four of the exceptional groups listed in Theorem 2.1 we denote by $H_{i}$ for $i \in\{1,2,3,4\}$; a precise representation of each of these groups is given in Theorem 2.1.

To represent some of our MRRs, we use cartesian products. For two graphs $\Gamma_{1}$ and $\Gamma_{2}$, the cartesian product of $\Gamma_{1}$ with $\Gamma_{2}$ is denoted by $\Gamma_{1} \square \Gamma_{2}$. It is the graph whose vertices are the elements of $V\left(\Gamma_{1}\right) \times V\left(\Gamma_{2}\right)$, with $\left(u_{1}, v_{1}\right)$ adjacent to $\left(u_{2}, v_{2}\right)$ if and only if either $u_{1}=u_{2}$ and $v_{1}$ is adjacent to $v_{2}$ in $\Gamma_{2}$, or $v_{1}=v_{2}$ and $u_{1}$ is adjacent to $u_{2}$ in $\Gamma_{1}$. We say that a graph $\Gamma$ on more than one vertex is prime with respect to the cartesian product if $\Gamma \cong \Gamma_{1} \square \Gamma_{2}$ implies that for some $i \in\{1,2\}, \Gamma_{i} \cong \Gamma$ and $\Gamma_{2-i}$ has just one vertex. It is well-known that every graph has a unique prime factorisation as the cartesian product of prime graphs. We say that two graphs are relatively prime with respect to the cartesian product if they have no common factors in their prime factorisations. We sometimes simply refer to the graphs as prime or relatively prime.

\section{Exceptional groups}

We begin by listing the 13 groups that do not admit a GRR but do not lie in either of the infinite families that do not admit GRRs.

The following theorem is the end result of considerable work by a number of researchers. Imrich [7] completed the abelian case (correcting an earlier error by Sabidussi [13] and Chao [2], who showed that no graph has a transitive abelian automorphism group, but overlooked the case of elementary abelian 2-groups). The construction given in [7] also has an error in the case of the elementary abelian 2-group of order 32; it is mentioned in [10] that this was pointed out and corrected by Alspach, Hell, Hetzel, and Lim, and a GRR for that group (due to Hetzel) appears in [10]. Watkins, alone and in joint work with Nowitz $[11,12,16,17,18]$ found the other ten exceptional groups and proved in [12] that any nonabelian group whose order is coprime to 6 admits a GRR. Imrich [8] then showed that every nonabelian group whose order is odd and at least $3^{7} \cdot 5^{4}$ admits a GRR. Hetzel [5, Satz 14.38] showed that the exceptions we have mentioned are the only solvable groups that fail to have GRRs, and Godsil [3] completed the result by showing that every nonsolvable group has a GRR. We therefore cite Godsil's work for the final result, but attribute it to all of the researchers who made major contributions.

Theorem 2.1 (Godsil, Hetzel, Imrich, Nowitz, and Watkins; see [3]). The following are the only groups that are neither generalised dicyclic nor abelian of exponent greater than 2, yet admit no GRR:

- $\mathbb{Z}_{2}^{2}, \mathbb{Z}_{2}^{3}, \mathbb{Z}_{2}^{4}$ 
- $D_{6}, D_{8}, D_{10}$ where these represent the dihedral groups of orders 6,8 , and 10 (respectively);

- $A_{4}$, the alternating group of degree 4;

- $H_{1}:=\left\langle a, b, c: a^{2}=b^{2}=c^{2}=1, a b c=b c a=c a b\right\rangle$;

- $H_{2}:=\left\langle a, b: a^{8}=b^{2}=1, b a b=a^{5}\right\rangle$;

- $H_{3}:=\left\langle a, b, c: a^{3}=b^{3}=c^{2}=1, a b=b a,(a c)^{2}=(b c)^{2}=1\right\rangle$;

- $H_{4}:=\left\langle a, b, c: a^{3}=c^{3}=1, a c=c a, b c=c b, b^{-1} a b=a c\right\rangle$;

- $Q_{8} \times \mathbb{Z}_{3}, Q_{8} \times \mathbb{Z}_{4}$, where $Q_{8}$ is the quaternion group of order 8 .

The groups listed in the first bullet are abelian, and their Cayley indices are given in Section 3.

All of the remaining groups have Cayley index 2. Their Cayley index must be at least 2 by Theorem 2.1, since they admit no GRR. This was shown explicitly in [16, Theorem 2]

Table 1: Cayley indices for all finite groups.

\begin{tabular}{|c|c|c|}
\hline Group & Cayley index & See \\
\hline \multicolumn{3}{|l|}{ Abelian groups } \\
\hline $\mathbb{Z}_{2}, \mathbb{Z}_{2}^{n}, n \geq 5$ & 1 & [7], 1.2 of [10] \\
\hline $\mathbb{Z}_{2}^{3}, \mathbb{Z}_{4} \times \mathbb{Z}_{2}$ & 6 & Lemma 2.7 of $[10]$ \\
\hline $\mathbb{Z}_{2}^{4}$ & 8 & Table 3 \\
\hline $\mathbb{Z}_{4}^{2}$ & 4 & Table 3 \\
\hline $\mathbb{Z}_{4} \times \mathbb{Z}_{2}^{2}$ & 8 & Table 3 \\
\hline $\mathbb{Z}_{3}^{2}$ & 8 & Lemma 2.4 of [10] \\
\hline $\mathbb{Z}_{3}^{3}$ & 12 & Table 3 \\
\hline all other abelian groups & 2 & Theorem 1 of [10] \\
\hline \multicolumn{3}{|l|}{ Hamiltonian 2-groups } \\
\hline$Q_{8}$ & 16 & Lemma 2.6 of [10] \\
\hline$Q_{8} \times \mathbb{Z}_{2}$ & 16 & Section 4 \\
\hline$Q_{8} \times \mathbb{Z}_{2}^{n}, n \geq 2$ & 8 & Proposition 4.8 \\
\hline \multicolumn{3}{|l|}{ Other generalised dicyclic groups } \\
\hline $\operatorname{Dic}\left(\mathbb{Z}_{6}, 3\right)$ & 4 & Table 4 \\
\hline $\operatorname{Dic}\left(\mathbb{Z}_{8}, 4\right)$ & 4 & Table 4 \\
\hline $\operatorname{Dic}\left(\mathbb{Z}_{10}, 5\right)$ & 4 & Table 4 \\
\hline $\operatorname{Dic}\left(\mathbb{Z}_{4} \times \mathbb{Z}_{2},(0,1)\right)$ & 4 & Table 4 \\
\hline all other generalised dicyclic groups & 2 & Section 5 , and Theorem 2 of [10] \\
\hline \multicolumn{3}{|l|}{ Exceptional groups } \\
\hline$D_{6}, D_{8}, D_{10}$ & 2 & Section 2 of [10], or Table 2 \\
\hline$A_{4}$ & 2 & Table 2 \\
\hline$Q_{8} \times \mathbb{Z}_{3}, Q_{8} \times \mathbb{Z}_{4}$ & 2 & Table 2 \\
\hline$H_{1}$ of order 16 & 2 & Table 2 \\
\hline$H_{2}$ of order 16 & 2 & Table 2 \\
\hline$H_{3}$ of order 18 & 2 & Table 2 \\
\hline$H_{4}$ of order 27 & 2 & Table 2 \\
\hline Every group not listed above & 1 & {$[3]$} \\
\hline
\end{tabular}


for the dihedral groups in the second bullet. It was shown in [18, Proposition 3.7] for $A_{4}$. For the groups $H_{1}$ and $H_{3}$, it was shown in [18, Proposition 5.3 and Theorem 2]. The group $H_{2}$ was dealt with in [11, Theorem 2 or Proposition 3.1], and $H_{4}$ in [12, Theorem 3]. Finally, $Q_{8} \times \mathbb{Z}_{3}$ and $Q_{8} \times \mathbb{Z}_{4}$ were addressed in [17, Theorem].

To show that the Cayley index of each is precisely 2, we present Table 2. For each group, we give the connection set for a Cayley graph on that group that has Cayley index 2. The Cayley indices of these graphs can be verified by hand or by computer.

Table 2: MRRs for exceptional groups.

\begin{tabular}{|c|c|}
\hline Group $G$ & $S$ such that $c(\operatorname{Cay}(G, S))=2$ \\
\hline $\begin{aligned} D_{2 n}=\left\langle a, b: a^{2}=b^{n}=1, a b a=\right. & \left.b^{-1}\right\rangle, \\
& n \in\{3,4,5\}\end{aligned}$ & $\{a, a b\}$ \\
\hline$A_{4}$ & $\left\{\left(\begin{array}{llll}1 & 2 & 3\end{array}\right)^{ \pm 1},\left(\begin{array}{lll}1 & 2\end{array}\right)\left(\begin{array}{lll}3 & 4\end{array}\right)\right\}$ \\
\hline $\begin{aligned} H_{1}=\left\langle a, b, c: a^{2}=b^{2}=c^{2}=1,\right. & \\
& a b c=b c a=c a b\rangle\end{aligned}$ & $\left\{a, b, c,(a b)^{ \pm 1}\right\}$ \\
\hline$H_{2}=\left\langle a, b: a^{8}=b^{2}=1, b a b=a^{5}\right\rangle$ & $\left\{a^{ \pm 1}, a^{ \pm 2}, b\right\}$ \\
\hline $\begin{aligned} H_{3}=\left\langle a, b, c: a^{3}=b^{3}=\right. & c^{2}=1, a b=b a, \\
& \left.(a c)^{2}=(b c)^{2}=e\right\rangle\end{aligned}$ & $\left\{a^{ \pm 1}, c, a c, b c\right\}$ \\
\hline $\begin{array}{r}H_{4}=\left\langle a, b, c: a^{3}=c^{3}=1, a c=c a, b c=c b,\right. \\
\left.b^{-1} a b=a c\right\rangle\end{array}$ & $\left\{a^{ \pm 1}, b^{ \pm 1},\left(a^{-1} b\right)^{ \pm 1},\left(b a b^{-1}\right)^{ \pm 1}\right\}$ \\
\hline$Q_{8} \times \mathbb{Z}_{3}=\left\langle i, j, z: z^{3}=1, i z=z i, j z=z j\right\rangle$ & $\left\{ \pm i,(i z)^{ \pm 1},(j z)^{ \pm 1}\right\}$ \\
\hline$Q_{8} \times \mathbb{Z}_{4}=\left\langle i, j, z: z^{4}=1, i z=z i, j z=z j\right\rangle$ & $\left\{z^{ \pm 1}, \pm i, \pm j,(i z)^{ \pm 1},(-k z)^{ \pm 1}\right\}$ \\
\hline
\end{tabular}

The MRRs listed in the first line of this table were also mentioned in [10].

\section{Abelian groups}

The Cayley index of every abelian group was determined in [10]. However, for a small number of these they stated only that the Cayley index had been found by Hetzel on computer, and cite a private communication. The known results on abelian groups are as follows.

Theorem 3.1 ([10, Theorem 1, Lemma 2.4, Lemma 2.7]). The only finite abelian groups with a Cayley index greater than 2 are:

- $\mathbb{Z}_{2}^{3}$ and $\mathbb{Z}_{4} \times \mathbb{Z}_{2}$, for which the Cayley index is 6 , with MRR $K_{2} \square K_{2} \square K_{2}$ (the cube);

- $\mathbb{Z}_{3}^{2}$, for which the Cayley index is 8 , with MRR $K_{3} \square K_{3}$;

- $\mathbb{Z}_{2}^{4}, \mathbb{Z}_{4} \times \mathbb{Z}_{2}^{2}$, and $\mathbb{Z}_{4}^{2}$; and

- $\mathbb{Z}_{3}^{3}$.

In the rest of this section, we list the Cayley index for each of the last four groups together with an MRR for each group. The Cayley indices for these graphs and the fact that these are the Cayley indices for these groups can be verified by computer. 
If $A$ is an abelian group that we are presenting as being isomorphic to $\mathbb{Z}_{i_{1}} \times \cdots \times \mathbb{Z}_{i_{k}}$, then we let $\left\{z_{1}, \ldots, z_{k}\right\}$ be the canonical generating set for this group, so $\left|z_{j}\right|=i_{j}$. We present the Cayley index and an MRR for each group in Table 3.

Table 3: MRRs for abelian groups not given in [10].

\begin{tabular}{c|c|c} 
Group & Cayley index & Connection set for an MRR \\
\hline \hline $\mathbb{Z}_{2}^{4}$ & 8 & $\left\{z_{1}, z_{2}, z_{3}, z_{4}, z_{1} z_{2}, z_{1} z_{3}, z_{2} z_{4}\right\}$ \\
$\mathbb{Z}_{4} \times \mathbb{Z}_{2}^{2}$ & 8 & $\left\{z_{1}^{ \pm 1}, z_{2}, z_{3},\left(z_{1} z_{2}\right)^{ \pm 1},\left(z_{1} z_{3}\right)^{ \pm 1}\right\}$ \\
$\mathbb{Z}_{4}^{2}$ & 4 & $\left\{z_{1}^{ \pm 1}, z_{2}^{ \pm 1}, z_{1}^{2},\left(z_{1} z_{2}\right)^{ \pm 1}\right\}$ \\
$\mathbb{Z}_{3}^{3}$ & 12 & $\left\{z_{1}^{ \pm 1}, z_{2}^{ \pm 1}, z_{3}^{ \pm 1},\left(z_{1} z_{2}\right)^{ \pm 1},\left(z_{1} z_{3}\right)^{ \pm 1},\left(z_{2} z_{3}\right)^{ \pm 1}\right\}$
\end{tabular}

It may seem odd that $c\left(\mathbb{Z}_{2}^{4}\right)>c\left(\mathbb{Z}_{2}^{3}\right)$. However, Lemma 4.4 does not apply here, because neither MRR for $\mathbb{Z}_{2}^{3}$ ( $K_{2} \square K_{2} \square K_{2}$ and its complement, $K_{4} \square K_{2}$ ), is relatively prime to $K_{2}$, which is the unique connected MRR for $\mathbb{Z}_{2}$.

\section{The groups $Q_{8} \times \mathbb{Z}_{2}^{n}$}

In this section we deal with a particular family of generalised dicyclic groups: groups of the form $Q_{8} \times \mathbb{Z}_{2}^{n}$ for some nonnegative integer $n$.

Definition 4.1. A hamiltonian group is a nonabelian group all of whose subgroups are normal. A hamiltonian 2-group is a hamiltonian group whose order is a power of 2 .

It is well-known (see, for example, [4, Theorem 12.5.4]) that the hamiltonian 2-groups are precisely the groups of the form $Q_{8} \times \mathbb{Z}_{2}^{n}$ for some nonnegative integer $n$ that we are considering in this section.

We begin with three important results from [10].

Lemma 4.2 ([10, Lemma 2.6]). The group $Q_{8}$ has Cayley index 16, with $C_{4} \square \overline{K_{2}}$ as an $M R R$.

Lemma 4.3 ([10, Proposition 2.9]). Every group other than $\mathbb{Z}_{2}^{2}, \mathbb{Z}_{2}^{3}, \mathbb{Z}_{4}, \mathbb{Z}_{4} \times \mathbb{Z}_{2}$, and $\mathbb{Z}_{3}^{2}$ admits a connected MRR that is prime with respect to the cartesian product.

Lemma 4.4 ([10, Lemma 2.8]). Let $G_{1}$ and $G_{2}$ be groups having connected MRRs that are relatively prime with respect to the cartesian product. Then $c\left(G_{1} \times G_{2}\right) \leq c\left(G_{1}\right) c\left(G_{2}\right)$.

In fact, if $\Gamma_{1}$ and $\Gamma_{2}$ are connected MRRs for $G_{1}$ and $G_{2}$ (respectively) that are relatively prime with respect to the cartesian product, then $c\left(\Gamma_{1} \square \Gamma_{2}\right)=c\left(G_{1}\right) c\left(G_{2}\right)$ and $\Gamma_{1} \square \Gamma_{2}$ is a Cayley graph on $G_{1} \times G_{2}$.

The following observation is made in [10] and is implicit in their Theorem 2(b), which states that $c\left(Q_{8} \times \mathbb{Z}_{2}^{n}\right) \leq 16$ for every integer $n \geq 0$. It can be deduced from Lemmas 4.2, 4.3, and 4.4, using the fact that $c\left(\mathbb{Z}_{2}\right)=1$.

Corollary 4.5. For every group $G \notin\left\{\mathbb{Z}_{2}^{2}, \mathbb{Z}_{2}^{3}, \mathbb{Z}_{4}, \mathbb{Z}_{4} \times \mathbb{Z}_{2}, \mathbb{Z}_{3}^{2}\right\}, c\left(G \times \mathbb{Z}_{2}\right) \leq c(G)$.

The following result is key to providing a lower bound for the Cayley index of every group $Q_{8} \times \mathbb{Z}_{2}^{n}$. 
Proposition 4.6 ([1, Classification Theorem $])$. There are 8 permutations $\varphi$ of the elements of $G=Q_{8} \times \mathbb{Z}_{2}^{n}$ that fix the identity, and have the property that for every $g, h \in G, \varphi(g h)$ is either $\varphi(g) h$, or $\varphi(g) h^{-1}$.

Corollary 4.7. The Cayley index of $Q_{8} \times \mathbb{Z}_{2}^{n}$ is at least 8 for every integer $n \geq 0$.

Proof. Fix $n$, and let $G=Q_{8} \times \mathbb{Z}_{2}^{n}$. Let $S$ be any inverse-closed subset of $G \backslash\left\{1_{G}\right\}$, and let $\Gamma=\operatorname{Cay}(G, S)$. Let $\varphi$ be any of the 8 permutations given in Proposition 4.6. To prove this result, it will be sufficient to show that $\varphi$ is an automorphism of $\Gamma$.

We know that for any $g \in G, g$ is adjacent to $g s$ if and only if $s \in S$. We also know that $\varphi(g s)$ is either $\varphi(g) s$, or $\varphi(g) s^{-1}$. Since $S$ is inverse-closed, each of these is adjacent to $\varphi(g)$ if and only if $s \in S$. Thus, $\varphi$ is indeed an automorphism of $\Gamma$.

To complete this section, we note that $\overline{C_{4} \square \overline{K_{2}}} \square K_{2}$ is an MRR for $Q_{8} \times \mathbb{Z}_{2}$ with Cayley index 16 , verified by computer. However, for $Q_{8} \times \mathbb{Z}_{2}^{2}$, the Cayley index is 8 , with $\operatorname{MRR} \operatorname{Cay}\left(Q_{8} \times \mathbb{Z}_{2}^{2},\left\{ \pm i, \pm j, \pm k, \pm i z_{1}, \pm k z_{1} z_{2}, z_{1}, z_{2}\right\}\right)$, where $z_{1}$ and $z_{2}$ are two distinct central involutions that do not lie in $Q_{8}$.

Thus, using Corollary 4.5 and Corollary 4.7 we are able to conclude the following.

Proposition 4.8. For every integer $n \geq 2$, the Cayley index of $Q_{8} \times \mathbb{Z}_{2}^{n}$ is 8 .

\section{Other generalised dicyclic groups}

Imrich and Watkins [10] showed that generalised dicyclic groups of order greater than 96 that are not of the form $Q_{8} \times \mathbb{Z}_{2}^{n}$ have Cayley index 2 . Many of the ideas from their proof in fact apply to generalised dicyclic groups of smaller orders. We reproduce these key ideas here, without their assumptions on order. We generally need to find two elements that satisfy a number of conditions. We note that the condition $a_{1} \neq y a_{2}$ was not listed in [10] but is required; for this reason we provide a full proof of Lemma 5.4.

Definition 5.1. Let $\operatorname{Dic}(A, y)$ be a generalised dicyclic group. We say that the 2 -set $\left\{a_{1}, a_{2}\right\}$ for $a_{1}, a_{2} \in A$ is a suitable pair of elements of $\operatorname{Dic}(A, y)$ if for every $\{i, j\}=$ $\{1,2\}$ we have

(i) $a_{1} \neq a_{2}, y a_{2}$;

(ii) $a_{i}^{2} \neq 1, y$;

(iii) $a_{i} \neq a_{j}^{2}, y a_{j}^{2}$; and

(iv) $a_{1} a_{2} \neq 1, y$.

Lemma 5.2. Let $D=\operatorname{Dic}\left(\langle z\rangle, z^{n}\right)$ (the dicyclic group of order $4 n$ ), where $|z|=2 n>10$. Then $\left\{z, z^{-2}\right\}$ is a suitable pair for $D$. If $\Gamma=\operatorname{Cay}\left(D,\left\{z^{ \pm 1}, x^{ \pm 1},(x z)^{ \pm 1},\left(x z^{-2}\right)^{ \pm 1}\right\}\right)$, where $x^{2}=z^{n}$, then $\langle z\rangle$ is invariant under $\operatorname{Aut}(\Gamma)_{1}$.

Proof. We have $y=z^{n}$. We verify the conditions for $\left\{z, z^{-2}\right\}$ to be a suitable pair. Since $n>5$, (i) and (ii) are satisfied; (iii) and (iv) are equally easy to check.

Let $\varphi \in \operatorname{Aut}(\Gamma)_{1}$ be arbitrary. It is straightforward to verify that when $n>4, z^{n}$ is the unique vertex that has 6 common neighbours with 1 , so $\varphi\left(z^{n}\right)=z^{n}$. In fact, this shows that for any vertex $v, v z^{n}$ is uniquely determined as the vertex that has 6 common neighbours with $v$. Since the neighbours of 1 can be partitioned into three pairs of this 
sort $\left(\left\{x, x^{-1}=x z^{n}\right\},\left\{x z, x z^{n+1}\right\}\right.$, and $\left.\left\{x z^{-2}, x z^{n-2}\right\}\right)$ and two elements $\left(z\right.$ and $\left.z^{-1}\right)$ whose match in this respect ( $z^{n+1}$, and $z^{n-1}$ respectively) is not a neighbour of 1 , it must be the case that $\left\{z, z^{-1}\right\}$ and $\left\{x, x^{-1}, x z, x z^{n+1}, x z^{-2}, x z^{n-2}\right\}$ are fixed setwise by $\varphi$. Repeating this argument shows that $\varphi\left(z^{i}\right) \in\langle z\rangle$ for every $i$. Thus, $\varphi(\langle z\rangle)=\langle z\rangle$.

Lemma 5.3. Let $A=\left\langle z_{1}, z_{2}\right\rangle$ where $\left|z_{1}\right|=2 n \geq 6,\left|z_{2}\right|=2$, and $z_{1} z_{2}=z_{2} z_{1}$, so $A \cong \mathbb{Z}_{2 n} \times \mathbb{Z}_{2}$. Then $\left\{z_{1}, z_{1}^{-2}\right\}$ is a suitable pair for $D=\operatorname{Dic}\left(A, x^{2}\right)$.

Also, if $\Gamma=\operatorname{Cay}\left(D,\left\{z_{1}^{ \pm 1}, z_{2}, x^{ \pm 1},\left(x z_{1}\right)^{ \pm 1},\left(x z_{1}^{-2}\right)^{ \pm 1}\right)\right.$ then $A$ is invariant under $\operatorname{Aut}(\Gamma)_{1}$.

Proof. Checking the conditions for $\left\{z_{1}, z_{1}^{-2}\right\}$ to be a suitable pair is straightforward.

Since $z_{2} \in S$ is central in $D$ and $x^{-1}=x z_{2}$, the following pairs of neighbours of 1 are adjacent in $\Gamma$ : $\left\{x, x^{-1}\right\} ;\left\{x z_{1}, x^{-1} z_{1}\right\} ;\left\{x z_{1}^{-2}, x^{-1} z_{1}^{-2}\right\}$. However, $z_{1}, z_{1}^{-1}$ and $z_{2}$ have no neighbours in $S$. Thus, we can distinguish the neighbours of 1 that lie in $A$ from the neighbours of 1 that lie in $x A$. Repeating this argument shows that every element of $A$ is invariant under $\operatorname{Aut}(\Gamma)_{1}$.

Lemma 5.4. Let $\Delta=\operatorname{Cay}(A, S)$ be an MRR of the abelian group A of Cayley index 2 . Let $D=\operatorname{Dic}(A, y)$ be a generalised dicyclic group with suitable pair $\left\{a_{1}, a_{2}\right\}$. Let

$$
\Gamma=\operatorname{Cay}\left(D, S \cup\left\{x, x^{-1}, x a_{1}, x^{-1} a_{1}, x a_{2}, x^{-1} a_{2}\right\}\right)
$$

(where $x$ is as in Definition 1.3) and suppose that for every $\varphi \in \operatorname{Aut}(\Gamma)_{1}$, we have $\varphi(A)=$ A. If $\varphi$ is not the identity automorphism, then $\varphi(a)=a$, and $\varphi(x a)=(x a)^{-1}$ for every $a \in A$.

Proof. Since $\varphi(A)=A$ and the induced subgraph on $A$ is $\Delta$ which has Cayley index 2, we know that we either have $\varphi(a)=a$ for every $a \in A$, or $\varphi(a)=a^{-1}$ for every $a \in A$. (This is always the case in a Cayley graph of Cayley index 2 on an abelian group.)

Similarly, since $\varphi(A)=A$ we have $\varphi(x A)=x A$. Observe that the induced subgraph on $x A$ is isomorphic to $\Delta$, which has Cayley index 2 . This means that there are exactly two graph automorphisms that fix $x A$ and take $x$ to any given vertex $x a$ where $a \in A$. Clearly one of these automorphisms is given by left-multiplication by $a^{-1}$, and therefore maps each vertex of the form $x a^{\prime}$ to the vertex $a^{-1} x a^{\prime}=x a a^{\prime}$. The other graph automorphism that fixes $x$ and $x A$ (aside from the identity) is the automorphism that maps every vertex $x a^{\prime}$ to the vertex $x\left(a^{\prime}\right)^{-1}$. This implies that the other automorphism that maps $x$ to $x a$ must take each vertex of the form $x a^{\prime}$ to the vertex $a^{-1} x\left(a^{\prime}\right)^{-1}=x a\left(a^{\prime}\right)^{-1}$.

In the remainder of this proof, we use $N_{X}(v)$ to denote the set of neighbours of the vertex $v$ that lie in the subset $X$ of the vertices of $\Gamma$. First we will show that $\varphi(x) \in$ $\left\{x, x^{-1}\right\}$.

We are assuming that $\varphi(A)=A$, and need to show that $\varphi(x) \notin\left\{x a_{1}, x^{-1} a_{1}, x a_{2}\right.$, $\left.x^{-1} a_{2}\right\}$. Suppose that $\varphi(x) \notin\left\{x, x^{-1}\right\}$. By symmetry, without loss of generality we may assume that $\varphi(x)=x a_{1}$.

Since $\varphi(x)=x a_{1}$ and $\varphi(x A)=x A$, as noted above we must have either $\varphi(x a)=$ $x a a_{1}$ for every $a \in A$, or $\varphi(x a)=x a^{-1} a_{1}$ for every $a \in A$.

Suppose the first of these possibilities holds, so $\varphi\left(x a_{1}\right)=x a_{1}^{2}$, which must therefore be a neighbour of 1 in $x A$, and hence an element of

$$
N_{x A}(1)=\left\{x, x^{-1}, x a_{1}, x^{-1} a_{1}, x a_{2}, x^{-1} a_{2}\right\} .
$$


Each of these possibilities contradicts one of the properties of being a suitable pair: any of the first four would contradict (ii); either of the last two contradict (iii).

If on the other hand the second possibility holds, then $\varphi\left(x a_{2}\right)=x a_{2}^{-1} a_{1} \in N_{x A}(1)$. Again, each possible equality contradicts one of the properties of being a suitable pair: either of the first two contradict (i); the third or fourth each contradicts (ii); and either of the last two contradict (iii). We therefore conclude that $\varphi(x) \in\left\{x, x^{-1}\right\}$, as claimed.

Next we show that $\varphi(a)=a$ for every $a \in A$.

Observe that

$$
N_{A}\left(x^{-1}\right)=N_{A}(x)=\left\{1, y, a_{1}, y a_{1}, a_{2}, y a_{2}\right\} .
$$

Thus, since $\varphi(x) \in\left\{x, x^{-1}\right\}$, we have $\varphi\left(N_{A}(x)\right)=N_{A}(x)$. If $\varphi(a)=a^{-1}$ for every $a \in A$, then this implies that $a_{1}^{-1} \in N_{A}(x)$, leading to a contradiction to the definition of a suitable pair, as above. (If $a_{1}^{-1}$ is any of the first four elements, this contradicts (ii); if it is either of the last two, this contradicts (iv).) Thus, we must have $\varphi(a)=a$ for every $a \in A$.

Next we show that if $\varphi(x)=x$ then $\varphi=1$.

Again as noted above, we must either have $\varphi(x a)=x a^{-1}$ for every $a \in A$, or $\varphi(x a)=$ $x a$ for every $a \in A$. In the latter case, $\varphi=1$ and we are done. In the former case, we must have $\varphi\left(N_{A}\left(x a_{1}^{-1}\right)\right)=N_{A}\left(x a_{1}\right)$. Observe that $a_{1}=x a_{1}^{-1} x^{-1} \in N_{A}\left(x a_{1}^{-1}\right)$, so this would imply that

$$
a_{1}=\varphi\left(a_{1}\right) \in N_{A}\left(x a_{1}\right)=\left\{a_{1}^{-1}, y a_{1}^{-1}, 1, y, a_{1}^{-1} a_{2}, y a_{1}^{-1} a_{2}\right\} .
$$

Similar to the arguments above, each of these possibilities contradicts some property of suitable pairs. If $a_{1}$ were any of the first four elements of $N_{A}\left(x a_{1}\right)$ this would contradict (i); if it were either of the last two, this would contradict (iii).

Finally, we show that if $\varphi(x)=x^{-1}$ then $\varphi(x a)=(x a)^{-1}$ for every $a \in A$.

Again as noted above, we must either have $\varphi(x a)=x^{-1} a=(x a)^{-1}$ for every $a \in A$, or $\varphi(x a)=x^{-1} a^{-1}$ for every $a \in A$. In the former case we are done. In the latter case, we must have $\varphi\left(N_{A}\left(x^{-1} a_{1}^{-1}\right)\right)=N_{A}\left(x a_{1}\right)$. Observe that $a_{1}=x^{-1} a_{1}^{-1} x \in N_{A}\left(x^{-1} a_{1}^{-1}\right)$, so this would imply that $a_{1}=\varphi\left(a_{1}\right) \in N_{A}\left(x a_{1}\right)$, yielding the same contradiction as in the previous paragraph.

Proposition 5.5. Let $A_{1}=\left\langle z_{1}\right\rangle$ be a cyclic group of order $2 n \geq 6$, and $A_{2}=\left\langle z_{1}, z_{2}\right\rangle$ with $\left|z_{2}\right|=2$ and $z_{1} z_{2}=z_{2} z_{1}$. Let $S_{1}=\left\{z_{1}, z_{1}^{-1}\right\}$ and $S_{2}=\left\{z_{1}, z_{1}^{-1}, z_{2}\right\}$, and let $D_{1}=\operatorname{Dic}\left(A_{1}, z_{1}^{n}\right)$, and $D_{2}=\operatorname{Dic}\left(A_{2}, z_{2}\right)$. Then

$$
\Gamma_{i}=\operatorname{Cay}\left(D_{i}, S_{i} \cup\left\{x, x^{-1}, x z_{1}, x z_{1}^{n+1}, x z_{1}^{-2}, x z_{1}^{n-2}\right\}\right)
$$

for $i \in\{1,2\}$ is connected and has Cayley index 2 when $n \geq 6$, and $\Gamma_{2}$ is connected and has Cayley index 2 when $n \geq 3$.

Proof. It is easy to see that $S_{1}$ is the connection set for a Cayley graph on $A_{1}$ with Cayley index 2. It is slightly less obvious that $S_{2}$ is the connection set for a Cayley graph on $A_{2}$ with Cayley index 2, but becomes clear upon noting that each $z_{1}$-edge lies in a unique 4cycle, while each $z_{2}$-edge lies in two 4 -cycles. Fix $i \in\{1,2\}$, and if $i=1$, ensure that $n \geq 5$.

By Lemma 5.2 or Lemma 5.3, we know that $\left\{z_{1}, z_{1}^{-2}\right\}$ is a suitable pair for $D_{i}$, and that for any $\varphi \in \operatorname{Aut}\left(\Gamma_{i}\right)_{1}, \varphi\left(A_{i}\right)=A_{i}$. By Lemma 5.4 with $S=S_{i}$ and this suitable pair, we see that there are only two possibilities for $\varphi: \varphi=1$, or $\varphi(a)=a$ and $\varphi(x a)=(x a)^{-1}$ for every $a \in A$. Thus, $\Gamma$ has Cayley index 2 . 
Proposition 5.6. Let $A$ be an abelian group of even order that contains an involution $y$, and let $D=\operatorname{Dic}(A, y)$. Suppose that $D$ has a connected MRR with Cayley index 2 . Let $A^{\prime}=A \times \mathbb{Z}_{2}$. Then $D^{\prime}=\operatorname{Dic}\left(A^{\prime}, y\right)$ has Cayley index 2 .

Proof. Observe that $D^{\prime} \cong D \times \mathbb{Z}_{2}$. The result is now immediate from Corollary 4.5.

As an immediate consequence of Proposition 5.5 and Proposition 5.6, we obtain the following.

Corollary 5.7. The following generalised dicyclic groups have Cayley index 2:

- $\operatorname{Dic}\left(A \times \mathbb{Z}_{2}^{k}, z_{1}^{n}\right)$ where $A=\left\langle z_{1}\right\rangle \cong \mathbb{Z}_{2 n}, n \geq 6$, and $k \geq 0$; and

- $\operatorname{Dic}\left(A \times \mathbb{Z}_{2}^{k}, z_{2}\right)$ where $A=\left\langle z_{1}, z_{2}\right\rangle \cong \mathbb{Z}_{2 n} \times \mathbb{Z}_{2},\left|z_{1}\right|=2 n,\left|z_{2}\right|=2, n \geq 3$, and $k \geq 0$.

This leads us to the following theorem.

Theorem 5.8. Every generalised dicyclic group that is neither abelian nor a hamiltonian 2-group has Cayley index 2, with the following four exceptions, each of which has Cayley index 4: $\operatorname{Dic}\left(\mathbb{Z}_{6}, 3\right)$, $\operatorname{Dic}\left(\mathbb{Z}_{8}, 4\right)$, $\operatorname{Dic}\left(\mathbb{Z}_{10}, 5\right)$, and $\operatorname{Dic}\left(\mathbb{Z}_{4} \times \mathbb{Z}_{2},(0,1)\right)$.

Proof. All generalised dicyclic groups of order greater than 96 have Cayley index of 2 (see [10]). To deal with the remaining cases, we begin by considering all abelian groups of even order at most 48 . For each group, we choose one representative for each automorphism class of elements of order 2 to be the distinguished element $y=x^{2}$.

By Corollary 5.7, the result holds for every dicyclic group of order at least 24; this deals with every cyclic group of even order at least 12 , all of which have a unique element of order 2 . Since $\mathbb{Z}_{2}$ produces an abelian dicyclic group and $\mathbb{Z}_{4}$ produces $Q_{8}$ which is a hamiltonian 2-group, we need only consider the dicyclic groups over the groups $\mathbb{Z}_{6}, \mathbb{Z}_{8}$, and $\mathbb{Z}_{10}$.

We note that if $n$ is odd, then $\mathbb{Z}_{2 n} \times \mathbb{Z}_{2}$ has only one automorphism class of elements of order 2, so that Corollary 5.7 provides an MRR for the unique generalised dicyclic group over any of these groups when $n \geq 3$, and in fact produces two MRRs when $n>6$. Also, if $n$ is even, $\mathbb{Z}_{2 n} \times \mathbb{Z}_{2}$ has two automorphism classes of elements of order 2 (the element $(n, 1)$ lies in the same class as $(0,1))$. Thus Corollary 5.7 produces an MRR for each of the two possible generalised dicyclic groups over these abelian groups whenever $n \geq 6$, and an MRR for one of them when $n \geq 3$. When $n=1$ there is a unique generalised dicyclic group which is actually abelian; and when $n=2$, one of the two generalised dicyclic groups is the hamiltonian 2-group $Q_{8} \times \mathbb{Z}_{2}$. Thus we need only consider the two groups $\operatorname{Dic}\left(\mathbb{Z}_{4} \times \mathbb{Z}_{2},(0,1)\right)$ and $\operatorname{Dic}\left(\mathbb{Z}_{8} \times \mathbb{Z}_{2},(4,0)\right)$.

The generalised dicyclic group over $\mathbb{Z}_{2}^{3}$ is abelian, and the groups

$$
\operatorname{Dic}\left(\mathbb{Z}_{4} \times \mathbb{Z}_{2}^{2},(2,0,0)\right) \cong Q_{8} \times \mathbb{Z}_{2}^{2} \quad \text { and } \quad \operatorname{Dic}\left(\mathbb{Z}_{4} \times \mathbb{Z}_{2}^{3},(2,0,0,0)\right)
$$

are hamiltonian 2-groups, so these need not be considered.

Finally, if a group has the form $D \times \mathbb{Z}_{2}$ for some smaller generalised dicyclic group $D$ with $c(D)=2$, then Corollary 4.5 gives $c\left(D \times \mathbb{Z}_{2}\right)=2$, so we do not have to consider these groups either. This eliminates all generalised dicyclic groups over $\mathbb{Z}_{6} \times \mathbb{Z}_{2}^{2}, \mathbb{Z}_{10} \times \mathbb{Z}_{2}^{2}$, and $\mathbb{Z}_{12} \times \mathbb{Z}_{2}^{2}$, as well as $\operatorname{Dic}\left(\mathbb{Z}_{8} \times \mathbb{Z}_{2}^{2},(0,1,0)\right)$. 
With all of this in mind, there are 18 generalised dicyclic groups that remain to be considered. We conclude this section and the paper with Table 4, showing the Cayley index and the connection set for an MRR for each of these generalised dicyclic groups. For four of these groups that have the form $D \times \mathbb{Z}_{2}$ for some smaller generalised dicyclic group $D$, we use Corollary 4.5 , but only after showing that $c(D)=2$ in a previous line of the table. For these, instead of explicitly giving the connection set for an MRR, we present the group as $D \times \mathbb{Z}_{2}$. This table completes the proof, and its results are straightforward to verify by computer.

Table 4: MRRs for generalised dicyclic groups.

\begin{tabular}{c|c|c} 
Group & $\begin{array}{c}\text { Cayley } \\
\text { index }\end{array}$ & Connection set for an MRR \\
\hline \hline $\operatorname{Dic}\left(\mathbb{Z}_{6}, 3\right)$ & 4 & $\left\{z_{1}^{ \pm 1}, x^{ \pm 1}\right\}$ \\
$\operatorname{Dic}\left(\mathbb{Z}_{8}, 4\right)$ & 4 & $\left\{z_{1}^{ \pm 1}, x^{ \pm 1}\right\}$ \\
$\operatorname{Dic}\left(\mathbb{Z}_{10}, 5\right)$ & 4 & $\left\{z_{1}^{ \pm 1}, x^{ \pm 1}\right\}$ \\
$\operatorname{Dic}\left(\mathbb{Z}_{4} \times \mathbb{Z}_{2},(0,1)\right)$ & 4 & $\left\{z_{1}^{ \pm 1}, x^{ \pm 1},\left(z_{1} x\right)^{ \pm 1}\right\}$ \\
$\operatorname{Dic}\left(\mathbb{Z}_{8} \times \mathbb{Z}_{2},(4,0)\right)$ & 2 & $\left\{z_{1}^{ \pm 1}, z_{2}, x^{ \pm 1},\left(z_{1} x\right)^{ \pm 1},\left(z_{2} x\right)^{ \pm 1}\right\}$ \\
$\operatorname{Dic}\left(\mathbb{Z}_{4} \times \mathbb{Z}_{4},(2,0)\right)$ & 2 & $\left\{z_{1}^{ \pm 1}, z_{2}^{ \pm 1},\left(z_{1} z_{2}\right)^{ \pm 1}, x^{ \pm 1},\left(z_{1} x\right)^{ \pm 1}\right\}$ \\
$\operatorname{Dic}\left(\mathbb{Z}_{4} \times \mathbb{Z}_{2}^{2},(0,1,0)\right)$ & 2 & $\left\{z_{1}^{ \pm 1}, z_{3}, x^{ \pm 1},\left(z_{1} x\right)^{ \pm 1},\left(z_{3} x\right)^{ \pm 1}\right\}$ \\
$\operatorname{Dic}\left(\mathbb{Z}_{6} \times \mathbb{Z}_{3},(3,0)\right)$ & 2 & $\left\{z_{1}^{ \pm 1}, z_{2}^{ \pm 1}, x^{ \pm 1},\left(z_{2} x\right)^{ \pm 1},\left(z_{1} z_{2} x\right)^{ \pm 1}\right\}$ \\
$\operatorname{Dic}\left(\mathbb{Z}_{8} \times \mathbb{Z}_{2}^{2},(4,0,0)\right)$ & 2 & $D \cong \operatorname{Dic}\left(\mathbb{Z}_{8} \times \mathbb{Z}_{2},(4,0)\right) \times \mathbb{Z}_{2}$ \\
$\operatorname{Dic}\left(\mathbb{Z}_{8} \times \mathbb{Z}_{4},(4,0)\right)$ & 2 & $\left\{z_{1}^{ \pm 1}, z_{2}^{ \pm 1}, x^{ \pm 1},\left(z_{1}^{6} z_{2}^{-1} x\right)^{ \pm 1},\left(z_{1}^{5} z_{2} x\right)^{ \pm 1}\right\}$ \\
$\operatorname{Dic}\left(\mathbb{Z}_{8} \times \mathbb{Z}_{4},(0,2)\right)$ & 2 & $\left\{z_{1}^{ \pm 1}, z_{2}^{ \pm 1}, x^{ \pm 1},\left(z_{1}^{5} x\right)^{ \pm 1},\left(z_{1}^{3} z_{2} x\right)^{ \pm 1}\right\}$ \\
$\operatorname{Dic}\left(\mathbb{Z}_{4}^{2} \times \mathbb{Z}_{2},(2,0,0)\right)$ & 2 & $D \cong \operatorname{Dic}\left(\mathbb{Z}_{4} \times \mathbb{Z}_{4},(2,0)\right) \times \mathbb{Z}_{2}$ \\
$\operatorname{Dic}\left(\mathbb{Z}_{4}^{2} \times \mathbb{Z}_{2},(0,0,1)\right)$ & 2 & $\left\{z_{1}^{ \pm 1}, z_{2}^{ \pm 1}, x^{ \pm 1},\left(z_{2}^{3} x\right)^{ \pm 1},\left(z_{1}^{3} z_{2}^{2} x\right)^{ \pm 1}\right\}$ \\
$\operatorname{Dic}\left(\mathbb{Z}_{4} \times \mathbb{Z}_{2}^{3},(0,1,0,0)\right)$ & 2 & $D \cong \operatorname{Dic}\left(\mathbb{Z}_{4} \times \mathbb{Z}_{2}^{2},(0,1,0)\right) \times \mathbb{Z}_{2}$ \\
$\operatorname{Dic}\left(\mathbb{Z}_{12} \times \mathbb{Z}_{3},(6,0)\right)$ & 2 & $\left\{z_{1}^{ \pm 1}, z_{2}^{ \pm 1}, x^{ \pm 1},\left(z_{1}^{7} z_{2} x\right)^{ \pm 1},\left(z_{1}^{3} z_{2} x\right)^{ \pm 1}\right\}$ \\
$\operatorname{Dic}\left(\mathbb{Z}_{6} \times \mathbb{Z}_{6},(3,0)\right)$ & 2 & $D \cong \operatorname{Dic}\left(\mathbb{Z}_{6} \times \mathbb{Z}_{3},(3,0)\right) \times \mathbb{Z}_{2}$ \\
$\operatorname{Dic}\left(\mathbb{Z}_{12} \times \mathbb{Z}_{4},(6,0)\right)$ & 2 & $\left\{z_{1}^{ \pm 1}, z_{2}^{ \pm 1}, x^{ \pm 1},\left(z_{1}^{4} z_{2} x\right)^{ \pm 1},\left(z_{1}^{9} z_{2}^{3} x\right)^{ \pm 1}\right\}$ \\
$\operatorname{Dic}\left(\mathbb{Z}_{12} \times \mathbb{Z}_{4},(0,2)\right)$ & 2 & $\left\{z_{1}^{ \pm 1},\left(z_{1}^{3} z_{2}\right)^{ \pm 1}, x^{ \pm 1},\left(z_{1} x\right)^{ \pm 1},\left(z_{1} z_{2} x\right)^{ \pm 1}\right\}$ \\
&
\end{tabular}

\section{References}

[1] D. P. Byrne, M. J. Donner and T. Q. Sibley, Groups of graphs of groups, Beitr. Algebra Geom. 54 (2013), 323-332, doi:10.1007/s13366-012-0093-7.

[2] C.-y. Chao, On a theorem of Sabidussi, Proc. Amer. Math. Soc. 15 (1964), 291-292, doi:10. $2307 / 2034055$.

[3] C. D. Godsil, GRRs for nonsolvable groups, in: L. Lovász and V. T. Sós (eds.), Algebraic Methods in Graph Theory, Volume I, North-Holland, Amsterdam, volume 25 of Colloquia Mathematica Societatis János Bolyai, pp. 221-239, 1981, papers from the conference held in Szeged, August 24 - 31, 1978. 
[4] M. Hall, Jr., The Theory of Groups, Macmillan, New York, 1959.

[5] D. Hetzel, Über reguläre graphische Darstellung von aufösbaren Gruppen, Diploma thesis, Technische Universität Berlin, Berlin, 1976.

[6] W. Imrich, Graphen mit transitiver Automorphismengruppe, Monatsh. Math. 73 (1969), 341347, doi:10.1007/bf01298984.

[7] W. Imrich, Graphs with transitive Abelian automorphism group, in: P. Erdős and A. Rényi (eds.), Combinatorial Theory and its Applications, Volume II, North-Holland, Amsterdam, volume 4 of Colloquia Mathematica Societatis János Bolyai, pp. 651-656, 1970, proceedings of the Colloquium on Combinatorial Theory and its Applications held at Balatonfüred, August 24 $-29,1969$.

[8] W. Imrich, On graphical regular representations of groups, in: A. Hajnal, R. Rado and V. T. Sós (eds.), Infinite and Finite Sets, Volume II, North-Holland, Amsterdam, volume 10 of Colloquia Mathematica Societatis János Bolyai, pp. 905-925, 1975, proceedings of a Colloquium held at Keszthely, June 25 - July 1, 1973 (dedicated to Paul Erdős on his 60th birthday).

[9] W. Imrich, On graphs with regular groups, J. Comb. Theory Ser. B 19 (1975), 174-180, doi: 10.1016/0095-8956(75)90082-9.

[10] W. Imrich and M. E. Watkins, On automorphism groups of Cayley graphs, Period. Math. Hungar. 7 (1976), 243-258, doi:10.1007/bf02017943.

[11] L. A. Nowitz and M. E. Watkins, Graphical regular representations of non-abelian groups, I, Canad. J. Math. 24 (1972), 993-1008, doi:10.4153/cjm-1972-101-5.

[12] L. A. Nowitz and M. E. Watkins, Graphical regular representations of non-abelian groups, II, Canad. J. Math. 24 (1972), 1009-1018, doi:10.4153/cjm-1972-102-3.

[13] G. Sabidussi, Vertex-transitive graphs, Monatsh. Math. 68 (1964), 426-438, doi:10.1007/ bf01304186.

[14] The GAP Group, GAP - Groups, Algorithms, and Programming, Version 4.8.6, 2016, http : //www.gap-system.org/.

[15] The Sage Developers, SageMath, the Sage Mathematics Software System (Version 7.5.1), 2017, http: //www. sagemath.org/.

[16] M. E. Watkins, On the action of non-Abelian groups on graphs, J. Comb. Theory Ser. B 11 (1971), 95-104, doi:10.1016/0095-8956(71)90019-0.

[17] M. E. Watkins, On graphical regular representations of $C_{n} \times Q$, in: Y. Alavi, D. R. Lick and A. T. White (eds.), Graph Theory and Applications, Springer, Berlin, volume 303 of Lecture Notes in Mathematics, pp. 305-311, 1972, proceedings of the Conference at Western Michigan University, Kalamazoo, Michigan, May 10 - 13, 1972 (dedicated to the memory of J. W. T. Youngs).

[18] M. E. Watkins, Graphical regular representations of alternating, symmetric, and miscellaneous small groups, Aequationes Math. 11 (1974), 40-50, doi:10.1007/bf01837731. 